This item was submitted to Loughborough's Research Repository by the author.

Items in Figshare are protected by copyright, with all rights reserved, unless otherwise indicated.

\title{
Symmetry-breaking and chaos in electron transport in semiconductor
}

\section{superlattices}

PLEASE CITE THE PUBLISHED VERSION

LICENCE

CC BY-NC-ND 4.0

\section{REPOSITORY RECORD}

Alekseev, Kirill N., E.H. Cannon, J.C. McKinney, F.V. Kusmartsev, and D.K. Campbell. 2019. "Symmetrybreaking and Chaos in Electron Transport in Semiconductor Superlattices". figshare.

https://hdl.handle.net/2134/1244. 


\title{
Symmetry-Breaking and Chaos in Electron Transport in Semiconductor Superlattices
}

\author{
Kirill N. Alekseev ${ }^{1,2,3}$, Ethan H. Cannon ${ }^{1}$, Jonathan C. McKinney ${ }^{1}$, Feodor V. Kusmartsev ${ }^{4}$, and D.K. Campbell ${ }^{1}$ \\ ${ }^{1}$ Department of Physics, University of Illinois at Urbana-Champaign, 1110 West Green St., Urbana, IL 61801, USA \\ ${ }^{2}$ Department of Physical Sciences, Box 3000, University of Oulu FIN-90014, Finland \\ ${ }^{3}$ Theory of Nonlinear Processes Laboratory, Kirensky Institute of Physics, Krasnoyarsk 660036, Russia \\ ${ }^{4}$ Department of Physics, Loughborough University, Loughborough LE11 3TU, UK
}

\begin{abstract}
We study the motion of ballistic electrons in a single miniband of a semiconductor superlattice driven by a terahertz laser polarized along the growth direction. We work in the semiclassical balance-equation model, including different elastic and inelastic scattering rates, and incorporating the self-consistent electric field generated by electron motion. We explore regions of complex dynamics, which can include chaotic behavior and symmetry-breaking. Finally, we estimate the magnitude of the DC current and voltage that spontaneously appear in regions of broken-symmetry for parameters characteristic of real semiconductor superlattices.
\end{abstract}

\section{INTRODUCTION}

Since the seminal work of Esaki and Tsu [1] predicting negative differential conductivity in the current-voltage characteristics of a semiconductor superlattice (SSL), SSLs have been the subject of intense theoretical and experimental study. SSLs consist of thin layers of alternating semiconductors - typically 10-100 layers ranging from 10 to $500 \AA$ in thickness - whose different band structures create a periodic potential along the growth axis, essentially forming a one-dimensional, artificial crystal. The superlattice period can be controlled during the growth process and is generally much larger than the period of the constituent materials. Consequently the conduction and valence bands break into narrow allowed and forbidden regions termed minibands, with typical widths ranging from 1 to $100 \mathrm{meV}$. For an introduction to SSL design and properties, see reference [2].

Recent experiments on SSLs in the presence of high-frequency fields have revealed many novel, nonlinear effects, including photon-assisted tunneling [3], a reduction of the current during laser irradiation [4], absolute negative conductivity [5], resonant increases in the current [6], and, most recently, deterministic chaos in the sequential resonant tunneling regime [7]. Our previous work [8] suggests that chaos should also occur in the miniband regime for a SSL driven by an AC field. In this brief note, we recall some of the principal results of that work and report our current studies of symmetry-breaking effects in electron transport. Using numerical simulations we establish the possibility of spontaneous DC current and voltage generation in response to a purely AC drive. Our results cover the whole range of frequency/amplitude of the applied field, extending a recent investigation of the high frequency, strong field limit $[9]$.

\section{BALANCE EQUATIONS}

We consider electrons subject to an external field $E_{\text {ext }}=E_{0} \cos (\Omega t)$ using the tight-binding dispersion relation, $\epsilon(k)=\frac{\Delta}{2}[1-\cos (k a)]$, for SSL period $a$, minibandwidth $\Delta$, electron energy $\epsilon$ and crystal momentum $k$. We assume that the frequency of the external field is not large enough to support Zener tunneling to higher minibands, and that the electric field is uniform across the SSL. To model the electron dynamics, we generalize the semiclassical balance equations of Ignatov et al. [10] by including the self-consistent electric field resulting from electron motion, obtaining $[8]$,

$$
\begin{aligned}
\dot{V} & =-e E_{t o t}(t) / m(\varepsilon)-\gamma_{v} V \\
\dot{\varepsilon} & =-e E_{t o t}(t) V-\gamma_{\varepsilon}\left(\varepsilon-\varepsilon_{0}\right) \\
\dot{E}_{s c} & =-4 \pi j / \kappa-\alpha E_{s c}
\end{aligned}
$$

where $E_{t o t}=E_{e x t}+E_{s c}$ is the total electric field, including the self-consistent contribution; $j=-e N V$ is the current density; $V(t)$ and $\varepsilon(t)$ are the average electron velocity and energy, respectively; $\gamma_{v}, \gamma_{\varepsilon}$, and $\alpha$ are phenomenological relaxation rates for the average velocity, average energy and self-consistent field, respectively; $\kappa$ is the average dielectric constant for the SSL; $\varepsilon_{0}$ is the temperature-dependent equilibrium energy; $N$ is the carrier concentration, assumed uniform across the SSL; and $m(\varepsilon)=\frac{m_{0}}{1-2 \varepsilon / \Delta}, m_{0}=\frac{2 \hbar}{\Delta a^{2}}$, is the effective mass of the electrons. 
The balance equations remain invariant under the symmetry transformation:

$$
t \rightarrow t+\frac{2 n+1}{2} T, V \rightarrow-V, E_{s c} \rightarrow-E_{s c}
$$

where $T$ is the period of the applied field and $n=0,1,2, \cdots ;$ in other words, if $\left\{V(t), \varepsilon(t), E_{s c}(t)\right\}$ satisfy (11)-(3), then so do $\left\{-V\left(t+\frac{2 n+1}{2} T\right), \varepsilon\left(t+\frac{2 n+1}{2} T\right),-E_{s c}\left(t+\frac{2 n+1}{2} T\right)\right\}$. Physically, this represents the lack of preferred spatial direction for a SSL without DC bias; under this transformation the electron velocity and the self-consistent field reverse direction, but the electron energy remains unaltered.

Symmetric solutions also remain invariant under transformation (四); hence $V\left(t+\frac{2 n+1}{2} T\right)=-V(t)$ and $E_{s c}(t+$ $\left.\frac{2 n+1}{2} T\right)=-E_{s c}(t)$. For such cases the time-averages of both the velocity and the self-consistent field are zero. However there may also exist solutions which do not satisfy this symmetry, for which $V$ and $E_{s c}$ have non-zero average values; these symmetry-broken solutions correspond to the spontaneous generation of a DC current and bias.

In the limiting case $\gamma_{v}=\gamma_{\varepsilon}=0$, (1)-(3) can be transformed to the equation describing the Resistively Shunted Junction (RSJ) model of a Josephson junction (JJ) [8,11] This model possesses a thoroughly studied symmetry analagous to (田) $[12,11]$. When biased by an AC current of frequency $\Omega$, the JJ may develop a DC component to its voltage difference satisfying the phase-locking condition $\langle U\rangle \propto n \Omega$, where $n$ is an integer, $U$ is the voltage across the $\mathrm{JJ}$, and angular brackets indicate time-averages [12].

\section{NUMERICAL STUDIES}

In our earlier work [8], we showed that the balance equations give rise to chaotic behavior for a wide range of damping and driving parameters. Readers should consult this reference for a full description of the numerics and scalings used in the simulations. Briefly, however, there are three important frequencies: the drive frequency, $\Omega$; the Stark frequency of the driving field, $\omega_{s}=e a E_{0} / \hbar$; and the frequency of the cooperative oscillations associated with the self-consistent field, $\omega_{E}=\sqrt{2 \pi e^{2} N a^{2} \Delta / \kappa \hbar^{2}}$ which is usually in the terahertz range. As it depends only on SSL material parameters, we use $\omega_{E}$ as the scale of inverse time, and throughout the rest of this article we consider the dimensionless frequencies $\Omega / \omega_{E}, \omega_{s} / \omega_{E}, \gamma_{v} / \omega_{E}, \gamma_{\varepsilon} / \omega_{E}$ and $\alpha / \omega_{E}$ without explicitly showing the scaling.

As in many studies of the JJ $[12,11]$, we examine the dynamics for fixed damping while varying the parameters of the applied field. Figures $1 \mathrm{a}$ and $1 \mathrm{~b}$ indicate the region of chaos and symmetry-breaking, respectively. Note the similar general shape of these regions; further work shows that for greater damping the region of chaos diminishes while the region of symmetry-breaking retains this general shape $[8,13]$.

Fig. 1c plots the time-averaged self-consistent field, $\left\langle e a E_{s c} / \hbar \omega_{E}\right\rangle$, as a function of external frequency, $\omega_{s}$, for the frequency $\Omega=0.9$. Phase-locked symmetry-breaking where $\left\langle e a E_{s c} / \hbar \omega_{E}\right\rangle \approx n \Omega / \omega_{E}$ for $n= \pm 1, \pm 2$ is clearly visible. A careful examination reveals that $\left\langle e a E_{s c} / \hbar \omega_{E}\right\rangle$ also depends weakly on $\omega_{s}$; therefore the phase-locking relation is only approximately satisfied.

From (3) it immediately follows that $\langle V\rangle \propto\left\langle E_{s c}\right\rangle$ for non-chaotic solutions. Consequently symmetry-breaking should be experimentally observable as a DC current and bias across an unbiased SSL illuminated by a terahertz laser. For representative SSL parameters of $a=100 \AA, N=3 \times 10^{15} \mathrm{~cm}^{-3}, \Delta=22 \mathrm{meV}$, and $\epsilon=13$ [6], $\omega_{E}=3.3 \times 10^{12} \mathrm{~s}^{-1}$. Taking $\left\langle e a E_{s c} / \hbar \omega_{E}\right\rangle_{\max }=1.5$ yields a DC bias per SSL period of $3 \mathrm{mV}$. Furthermore, a lateral area of $8 \mu m^{2}$ would result in a current of $1 \mu \mathrm{A}$. Both these magnitudes should be experimentally detectable.

\section{CONCLUSION}

We have studied the complex dynamics of semiclassical balance equations that model the motion of electrons in the miniband of a semiconductor superlattice exposed to an external ac field. The equations include the self-consistent field created by electron motion. Numerical simulations indicate the possibility of broken-symmetry solutions, which occur in roughly the same laser frequency and electric field ranges as chaotic dynamics. The symmetry-breaking engenders a DC bias and current that could be experimentally detectable.

We would like to contrast this work with recent theoretical [14] and experimental [7] work predicting and observing chaos for SSLs in the incoherent tunneling regime when the electric field breaks into domains of different magnitudes [2]. Our model considers the coherent motion of electrons within a miniband in a spatially homogenous electric field. A complete theoretical understanding of when the applied field breaks into domains has not yet been attained, so it is 
encouraging that chaos is predicted in quite different models applicable in different regimes of SSL design and applied field characteristics.

We are especially grateful to Gennady Berman for his advice and continuing collaboration on many aspects of chaos in mesoscopic systems. We thank Sergei Turovets for a discussion on the notation of symmetry-breaking in dynamical systems. K.N.A. thanks the Department of Physics at The University of Illinois at Urbana-Champaign for hospitality. This work was partially supported by Linkage Grant No. 93-1602 from the NATO Special Programme Panel on Nanotechnology. K.N.A. was supported by INTAS(94-2058) and KRSF(6F0030). E.H.C. thanks the U.S. Department of Education for support by a GAANN Fellowship (DE-P200A40532), and J.C.M. thanks the U.S.-NSF for support under its REU program (NSF Grant No. PHYS93-22320).

* also at Landau Institute for Theoretical Physics, Moscow 142432, Russia

[1] L. Esaki and R. Tsu. Superlattice and negative differential conductivity in semiconductors. IBM J. Res. Dev., 14:61, 1970.

[2] H. T. Grahn, editor. Semiconductor Superlattices: Growth and Electronic Properties. World Scientific, New Jersey, 1995.

[3] B. J. Keay, S. J. Allen, Jr., J. Galán, J. P. Kaminski, K. L. Campman, A. C. Gossard, U. Bhattacharya, and M. J. W. Rodwell. Photon-assisted electric field domains and multiphoton-assisted tunneling in semiconductor superlattices. Phys. Rev. Lett., 75:4098, 1995.

[4] E. Schomburg, A. A. Ignatov, J. Grenzer, K. F. Renk, D. G. Pavel'ev, Yu. Koschurinov, B. Ja. Melzer, S. Ivanov, S. Schaposchnikov, and P. S. Kop'ev. Suppression of current through as Esaki-Tsu GaAs/AlAs superlattice by millimeter wave irradiation. Appl. Phys. Lett., 68:1096, 1996.

[5] B. J. Keay, S. Zeuner, S. J. Allen, Jr., K. D. Maranowski, A. C. Gossard, U. Bhattacharya, and M. J. W. Rodwell. Dynamic localization, absolute negative conductance, and stimulated, multiphoton emission in sequential resonant tunneling semiconductor superlattices. Phys. Rev. Lett., 75:4102, 1995.

[6] K. Unterrainer, B. J. Keay, M. C. Wanke, S. J. Allen, D. Leonard, G. Medeiros-Ribeiro, U. Bhattacharya, and M. J. W. Rodwell. Inverse Bloch oscillator: Strong terahertz-photocurrent resonances at the Bloch frequency. Phys. Rev. Lett., 76:2973, 1996.

[7] Y. Zhang, J. Kastrup, R. Klann, K. H. Ploog, and H. T. Grahn. Synchronization and chaos induced by resonant tunneling in GaAs/AlAs superlattices. Phys. Rev. Lett., 77:3001, 1996.

[8] K. N. Alekseev, G. P. Berman, D. K. Campbell, E. H. Cannon, and M. C. Cargo. Dissipative chaos in semiconductor superlattices. Phys. Rev. B, 54:10625, 1996.

[9] A. A. Ignatov, E. Schomburg, J. Grenzer, K. F. Renk, and E. P. Dodin. THz-field induced nonlinear transport and dc voltage generation in a semiconductor superlattice due to Bloch oscillations. Z. Phys. B, 98:187, 1995.

[10] A. A. Ignatov, K. F. Renk, and E. P. Dodin. Esaki-Tsu superlattice oscillator: Josephson-like dynamics of carriers. Phys. Rev. Lett., 70:1996, 1993.

[11] A. H. MacDonald and M. Plischke. Study of the driven damped pendulum: Applications to Josephson junctions and charge-density-wave systems. Phys. Rev. B, 27:201, 1983.

[12] D. D'Humieres, M. R. Beasley, B. A. Huberman, and A. Libchaber. Chaotic states and routes to chaos in the forced pendulum. Phys. Rev. A, 26:3483, 1982.

[13] K. N. Alekseev, E. H. Cannon, J. C. McKinney, F. V. Kusmartsev, and D. K. Campbell. Spontaneous dc current generation in resistively shunted semiconductor superlattices driven by a teraHertz field. in preparation. [See: Phys. Rev. Lett., 80:2669, 1998].

[14] O. M. Bulashenko, M. J. García, and L. L. Bonilla. Chaotic dynamics of electric-field domains in periodically driven superlattices. Phys. Rev. B, 53:10008, 1996. 


$$
\gamma_{\varepsilon}=.01 \gamma_{\mathrm{v}}=.01 \alpha=.001
$$

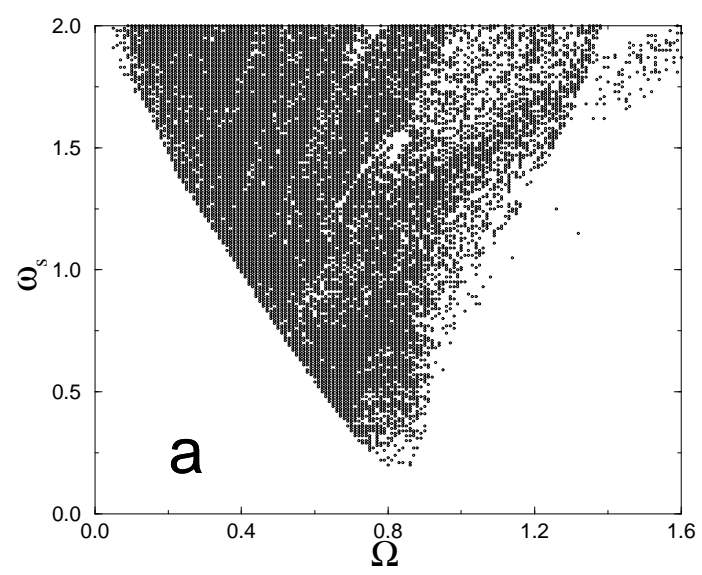

$$
\gamma_{\varepsilon}=.01 \gamma_{\mathrm{v}}=.01 \alpha=.001
$$

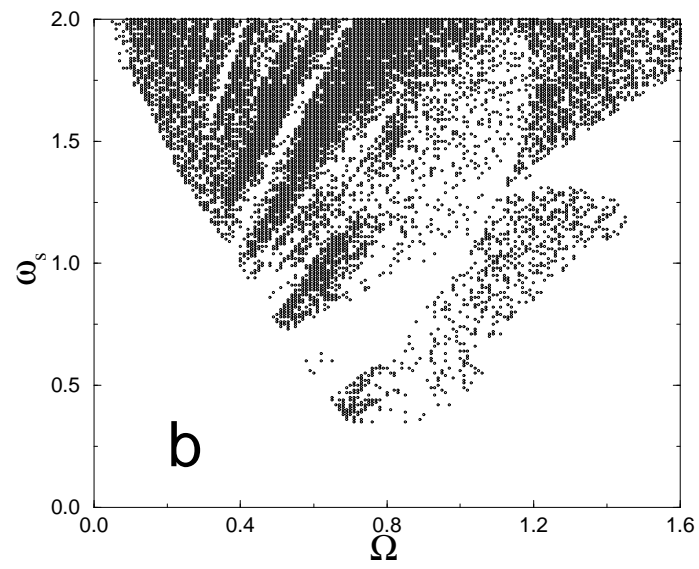

$$
\gamma_{\varepsilon}=.01 \gamma_{v}=.01 \alpha=.001 \Omega=.9
$$

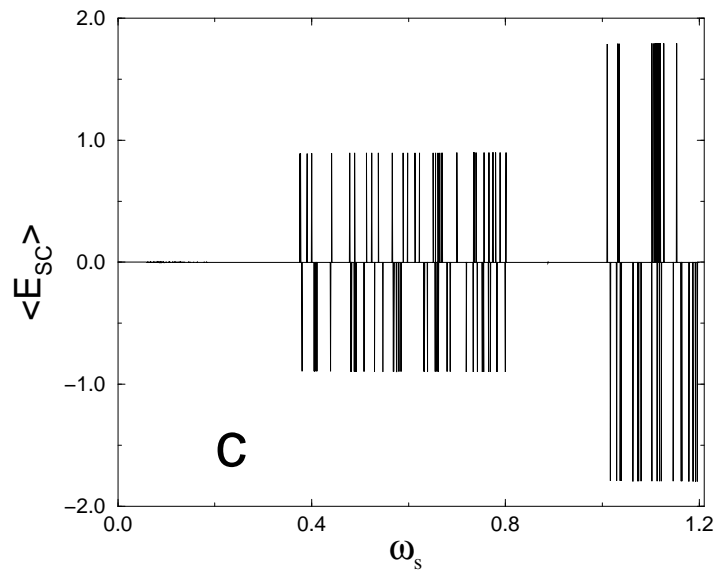

FIG. 1. For a weakly damped SSL, chaotic (dark symbols) and regular (white areas) regions (a); symmetry-broken (dark symbols) and symmetric (white areas) regions (b); time-averaged self-consistent field as a function of applied field strength (c) 